\title{
Pressemitteilungen und Stellungnahmen
}

\author{
http://www.djb.de/stellungnahmen-und-pressemitteilungen/
}

\section{Pressemitteilungen}

09-02 Mehr Gerechtigkeit im Alter nach der Scheidung: djb sorgte im Bundestag für Korrekturen bei Reform des Versorgungsausgleichs. (Februar 2009)

09-01 Deutsche Gleichstellungs- und Frauenpolitik bei der UN verhandelt - Der Deutsche Juristinnenbund (djb) zieht Bilanz. (Januar 2009)

08-19 CEDAW-Alternativbericht der Allianz deutscher Frauenverbände zum 6. Bericht der Bundesregierung an den CEDAW-Ausschuss. (Dezember 2008)

08-18 djb begrüßt Intention der Bundesregierung, Leistungen für Familien zu verbessern. (November 2008)

08-17 Zur Reform der Juristenausbildung. (November 2008)

08-16 Elterngeld ist für Eltern bei Teilzeitarbeit unattraktiv. Der Deutsche Juristinnenbund (djb) unterstützt den Offenen Brief zum Bundeselterngeldgesetz, in dem zahlreiche Verbände und Expert/innen Nachbesserungen fordern. (November 2008)

08-15 Einführung eines optionalen „Faktorverfahrens“ ist gleichstellungspolitisch nur eine scheinbare Verbesserung der Ehebesteuerung. (Oktober 2008)

08-14 Zum neuen Verfahren im Familienrecht. (Oktober 2008)

08-13 Der Deutsche Juristinnenbund feiert im Deutschen Bundestag sein 60-jähriges Bestehen. (September 2008)

08-12 Juristinnen unterstützen Elterngeld. (September 2008)

\section{Stellungnahmen}

09-01 „Entgeltgleichheit zwischen Frauen und Männern“. Stellungnahme zur öffentlichen Anhörung im Ausschuss für Familie, Senioren, Frauen und Jugend des Deutschen Bundestages am Mittwoch, den 28. Januar 2009. (Januar 2009)

08-24 Stellungnahme zum Referentenentwurf eines Bundesgesetzes zur Verbesserung des Kinderschutzes (Bundeskinderschutzgesetz - BkiSchG). (Dezember 2008)

08-23 Supplementary Shadow Report by the Deutscher Juristinnenbund (German Women Lawyers Association) in response to the List of issues and questions from the CEDAW committee's 43rd presession concerning the 6th Periodic Report by the German Government on the United Nations
Convention on the Elimination of All Forms of Discrimination against Women (CEDAW). (Dezember 2008)

08-22 Entwurf eines Niedersächsischen Gleichberechtigungsgesetzes (NGG) - Gesetzentwurf der Fraktionen der CDU und der FDP - Drs. 16/281. (November 2008)

08-21 Stellungnahme zu dem Gesetzentwurf der Bundesregierung „Entwurf eines Gesetzes zur Förderung von Familien und haushaltsnahen Dienstleistungen (Familienleistungsgesetz - FamLeistG) “ - BT-Drucksache 16/10809 - sowie zur Stellungnahme des Bundesrates - BR-Drucksache 753/08 -anlässlich der Anhörung im Finanzausschuss des Deutschen Bundestages am Montag, den 24. November 2008. (November 2008)

08-20 Offener Brief an die Konferenz der Justizministerinnen und Justizminister am 20. November 2008 in Berlin- zur Reform der Juristenausbildung -. (Oktober 2008)

08-19 Stellungnahme im Rahmen der Öffentlichen Anhörung des Familienausschusses des Deutschen Bundestages am 12. November 2008 zur Möglichkeit der bundeseinheitlichen Finanzierung von Frauenschutzhäusern. (Oktober 2008)

08-18 Stellungnahme des Landesverbands Hamburg im Deutschen Juristinnenbund (djb) zum SterniParkUrteil des Hamburgischen Oberverwaltungsgerichts. (Oktober 2008)

08-17 Stellungnahme des Landesverbands Hamburg zur Novelle des Hamburgischen Richtergesetzes. (Oktober 2008)

08-16 Stellungnahme zum Entwurf eines Gesetzes zur Änderung des Erb- und Verjährungsrechts BTDrucksache 16/8954 - anlässlich der Anhörung im Rechtsausschuss des Deutschen Bundestages am Mittwoch, den 8. Oktober 2008. (Oktober 2008)

08-15 Stellungnahme zum Entwurf der Bundesregierung zum Jahressteuergesetz 2009 (JStG 2009) (BT-Drs. 16/10189). Einführung eines optionalen „Faktorverfahrens“ für die Lohnsteuer bei Ehegatten, $\mathbb{S} 39 \mathrm{f}$ EStG - neu -. (September 2008)

08-14 Stellungnahme zum Abkommensentwurf zum deutsch-französischen Wahlgüterstand. (September 2008)

08-13 Stellungnahme zum Entwurf eines Gesetzes zur Änderung des Bundeselterngeld- und Elternzeitgesetzes (BT-Drucksache 16/9415). (September 2008, 6 Seiten) 